\title{
LA VERIFICABILIDAD DEL LENGUAJE RELIGIOSO: UN ANÁLISIS FILOSÓFICO
}

\section{THE VERIFIABILITY OF RELIGIOUS LANGUAGE: A PHILOSOPHICAL ANALYSIS}

\section{Damían Islas Mondragón ${ }^{1}$ Universidad Nacional Autónoma de México (México)}

Resumen: Las experiencias místicas y el conocimiento que producen son comunicados a través de un lenguaje religioso. En este texto presentaré un estudio de caso de una tradición yóguica del norte de la India, el Surat Shabdt Yoga, que se practica para obtener este tipo de experiencia. Desde el punto de vista de la teoría verificacionista del significado desarrollada por Alfred J. Ayer, el lenguaje religioso carece de significado. Mi objetivo es analizar y evaluar la teoría verificacionista y mostrar que no sólo se auto refuta; sino que también exhibe ciertas inconsistencias lógicas, empíricas y cognitivas que la debilitan como un buen criterio para evaluar la significatividad del lenguaje religioso. Se argumentará que los instrumentos y métodos de verificación se constriñen a nuestras habilidades, límites, expectativas e intereses cognitivos específicos; por lo que el valor de verdad del contenido fáctico de los enunciados religiosos no puede ser decidido de forma indubitable.

Palabras clave: EXPERIENCIA MÍSTICA; YOGA; LENGUAJE RELIGIOSO; VERIFICABILIDAD EMPÍRICA

\begin{abstract}
Mystical experiences and the knowledge they produce are communicated through a religious language. In this text I will present a case study of a yogic tradition in northern India, Surat Shabdt Yoga, which is practiced in order to obtain this type of experience. From the point of view of the verificationist theory of meaning developed by Alfred J. Ayer, the religious language has no meaning. My objective is to analyze and evaluate the verification theory and show that it not only refutes itself; it also exhibits certain logical, empirical and cognitive inconsistencies that weaken it as a good criterion to evaluate the significance of religious language. I will argue that instruments and methods of verification are constrained to our specific abilities, limits, expectations
\end{abstract}

[1] (damianislas@ujed.mx) es, desde 2010, Doctor en Filosofía de la Ciencia por la Universidad Autónoma Metropolitana (UAM). Ha ejercido como profesor de tiempo completo en la Universidad Juárez del Estado de Durango (UJED) donde, además, se ha desempeñado, de 2011 a 2012, como Coordinador académico de la Maestría en Ciencias y Humanidades. Ejerce, actualmente, como profesor adscrito al Centro Peninsular en Humanidades y en Ciencias Sociales de la UNAM. 
and cognitive interests; so that the truth value of the factual content of religious statements cannot be decided undoubtedly.

Key words: MYSTICAL EXPERIENCIE; YOGA; RELIGIOUS LANGUAGE; EMPIRICAL VERIFIABILITY

\section{Introducción}

De acuerdo con la teoría verificacionista del significado desarrollada por Alfred Jules Ayer, cualquier tipo de lenguaje es significativo si y sólo si (a) es analíticamente verdadero, esto es, si es verdadero en virtud del significado de sus términos constitutivos o b) exhibe cierto contenido factual empíricamente verificable. El argumento de que el lenguaje religioso carece de significado ha sido tan ampliamente aceptado, que para muchos autores se ha convertido en uno de los principales retos que ha enfrentado la filosofía de la religión pasada y presente (Martin, 2010, 458-466 y Wierenga, 1997, 295). No obstante que en la actualidad es generalmente aceptado que la teoría verificacionista se auto refuta, el presente estudio tiene como objetivo central evaluar la teoría verificacionista y mostrar que esta teoría no sólo se auto refuta; sino que también exhibe ciertas inconsistencias lógicas, empíricas y cognitivas que la debilitan como un buen criterio para evaluar la significatividad del lenguaje religioso.

Para contextualizar el tema, presentaré un estudio de caso de una tradición yóguica del norte de la India, el Surat Shabdt Yoga, que se practica para obtener una experiencia mística. Tanto la experiencia mística misma, como el conocimiento adquirido mediante de esta práctica yóguica, son expresados y comunicados utilizando un lenguaje religioso. De acuerdo con Ayer, no existe nada excepcional en el lenguaje religioso que nos impida analizarlo de manera similar a cualquier otro tipo de lenguaje distinto al religioso, como son el lenguaje científico o el lenguaje histórico. Y dado que los enunciados utilizados en el lenguaje religioso no son analíticamente verdaderos ni empíricamente verificables, se argumenta que carecen de valor lingüístico al estar constituidos de pseudo conceptos sin significado. Veamos un ejemplo. La afirmación judeo-cristiana:

(1) Dios es omnisciente

Exhibe varias características desde un punto de vista filosófico, a saber:

(a) La oración (1) expresa la creencia en que Dios es omnisciente.

(b) El estado psicológico de 'creer' que Dios es omnisciente no es ni verdadero ni falso. Lo que es susceptible de ser verdadero o falso es el contenido proposicional de esta oración, esto es, 'que Dios es omnisciente'. 
(c) El contenido proposicional de (1), esto es, 'que Dios es omnisciente'; es verdadero o falso independientemente de nuestras creencias.

(d) $\mathrm{Si}$ (1) es una oración que representa (de manera verdadera o falsa) un hecho o estado de cosas (el hecho de que Dios es omnisciente); presumiblemente no existe nada excepcional en (1) que nos impida analizarla de manera similar a cualquier otra oración perteneciente a tipos de lenguaje distintos al religioso como el lenguaje científico o el lenguaje histórico.

En este estudio partimos de la premisa de que las proposiciones lingüísticas de índole religioso expresan todo tipo de realidades, entidades, agentes, estados de cosas o propiedades como Dios, ángeles, milagros, salvación, iluminación, santidad, gracia, etc. De manera que la afirmación (1) «Dios es omnisciente», puede ser interpretada como una oración descriptiva que expresa, en este caso, la propiedad de un Ente. El análisis de la teoría verificacionista del significado desarrollada por Ayer nos permitirá evaluar las consecuencias filosóficas que tiene su teoría en el análisis del lenguaje religioso con el que se afirman proposiciones lingüísticas de este tipo, por lo que en este estudio dejaremos de lado las proposiciones religiosas que no pretenden constituirse en oraciones descriptivas literales como son las metáforas, las historias de ficción o la oración. Concluiré mostrando que las inconsistencias intrínsecas que exhibe la teoría verificacionista de Ayer, la debilitan como un buen criterio para evaluar la significatividad no sólo del lenguaje religioso; sino de cualquier otro lenguaje.

\section{El Surat Shabdt Yoga}

El contenido religioso de las experiencias místicas dependen, en cierto grado, de la tradición religiosa de la que se trate. Dado que existen diversas tradiciones religiosas a lo largo de la historia del ser humano, los conceptos de "contenido religioso» y "experiencia mística» son conceptos polisémicos. Basados en el carácter polisémico del lenguaje religioso, algunos autores han trazado una diferencia categórica entre las experiencias religiosas y las experiencias místicas argumentando que todas las experiencias místicas se constituyen en experiencias religiosas; pero que no todas las experiencias religiosas se constituyen en experiencias místicas.

De acuerdo con Jerome Gellman, las experiencias místicas pueden ser clasificadas en dos tipos principales, a saber, (a) una súper percepción sensorial o (b) una sub percepción sensorial. Ambas nos conducirían a la percepción de realidades o estado de cosas que no son accesibles a través de la percepción sensorial directa ni a través de un ejercicio de introspección normal (Gellman, 2005, 138). 
Ciertamente, si entendemos el misticismo como una práctica religiosa cuyo objetivo es la realización o la iluminación del individuo a través de la cual se aspira a ser uno con la divinidad o con la realidad última, entonces la experiencia religiosa constituye una categoría más amplia que el misticismo dado que muchas experiencias religiosas, como las vividas por algunos profetas como Hermes, Krishna, Moisés, Zoroastro o Mahoma, presumiblemente no se lograron como resultado de una práctica deliberada para producir la iluminación. Sin embargo, existen ciertas prácticas religiosas conducidas para alcanzar la unidad con Dios. Tal es el caso del Surat Shabdt Yoga.

De acuerdo con esta tradición yóguica de la comunidad Sij del norte de la India, la palabra "religión» proviene del latín 'religio' conformado por el prefijo 're' (intensidad), el verbo 'ligare' (ligar, unir) y el sufijo 'ion' (acción), esto es, la acción que liga o reúne intensamente. Esta reunión del atma (alma individual) con el paramatma (alma más alta) suele ser interpretada como la reunión del individuo con la divinidad (Swami 1983). El yoga, del sánscrito yug que significa «acoplar» o «ligar», es la práctica religiosa a través de la cual un individuo busca dominar su energía psíquica a partir de la más intensa concentración mental que impulsa el paso de las limitaciones del yo individual hacia la personalidad trascendente. En este sentido, la práctica yóguica busca apartar la conciencia individual de su "prisión» sensorial con el fin de alcanzar una integración con el todo.

Sabemos que la tradición yóguica ya estaba presente en los Vedas, una de las colecciones de libros sagrados más importantes para el Hinduísmo. De acuerdo con los Vedas, el yogui debe pasar por cuatro "ashrams» o estados llamados Brahmacharya, Grihasta, Vanprastha y Sannyasa. Cada uno de estos estados yóguicos constituyen, respectivamente, un período de la vida. El primer período es la preparación y aprendizaje durante el cual se estudia y se permanece célibe. El segundo período es el matrimonio y el desarrollo de la vida mundana. El tercer período es de retiro espiritual. El cuarto y último período es la renuncia definitiva del mundo físico. Cada una de estas etapas de la vida es considerada una preparación para la etapa siguiente que culmina cuando el Yo individual logra sumergirse o identificarse con el Uno absoluto. Así entendida, toda la vida es yoga.

El camino correcto es el "sádhana» (disciplina y práctica espiritual) el cual conduce a la consecución de la más importante meta de la vida, a saber, el «mukti» o liberación de la esclavitud del mundo. Los hindúes creen que tarde o temprano todo ser vivo está destinado a alcanzar el mukti. Sin embargo, el mukti no puede realizarse si no es a través de la reencarnación en la rueda de la vida o samsara. Para alcanzar el mukti, el atma individual debe «contactar» con el paramatma y, así, encontrar la 
«verdad» a través de la conciencia. El «dhyana» o meditación yóguica se realiza en tres etapas fundamentales, a saber, (i) la concentración, (ii) la meditación propiamente dicha y (iii) la contemplación. La práctica yóguica del Surat Shabdt Yoga (del punjabi surat «música» y shabdt «corriente o río»), busca detener todo proceso del pensamiento. Si la mente se tranquiliza de una manera total, se adquiere la habilidad para percibir la realidad como un todo. Asimismo, se logra controlar al cuerpo hasta el punto en el que ningún estímulo o agitación externo puede perturbar la mente. De esta forma, la mente del yogui no duerme y permanece atenta a las más sutiles percepciones de la conciencia.

Algunos estudiosos del tema consideran que la práctica yóguica comenzó con Patanjali, quien estableció lo que hoy se conoce como Raja-Yoga. En realidad, Patanjali sólo sistematizó las prácticas espirituales esparcidas ya en diversos tratados de su época como los Upanishadas. Los «rishis» védicos eran yoguis especializados que practicaron varios tipos de yoga, a saber, el yoga-sutra; el raja-yoga; el bhadti-yoga; el gnana-yoga; el karma-yoga; el nada-yoga; el hatha-yoga; el kundalini-yoga; el laya-yoga; el kriya-yoga; el mantra-yoga; el nama-yoga; el maha-yoga; el japa-yoga, entre otros. De manera que existe una multiplicidad de caminos yóguicos para la obtención de una experiencia mística. No obstante, podemos afirmar que el objetivo de cada uno de estos caminos yóguicos es el mismo, esto es, el auto-descubrimiento y la purificación para poder reunirse - religarse - con Dios y lograr la liberación de los «ikas» (ataduras de la existencia).

Dada esta multiplicidad de tradiciones yóguicas, cabe preguntarse cómo podemos elegir el «mejor» de los caminos yóguicos. Para ello, algunas tradiciones aconsejan la guía de un "gurú» experimentado. El gurú es aquel que puede revelar la verdad y liberar al individuo. El gurú es quien inicia al discípulo en el "sádhana» y sólo a través de su dirección se alcanza el «siddhi», esto es, el éxito, la realización y la perfección yóguica. Por lo tanto, la fe y obediencia al gurú parecen ser elementos esenciales en el sádhana yóguico.

No obstante que la tradición del gurú se remonta al pasado distante, en la actualidad forma parte integral del patrón educativo y religioso de la India. Hay quienes consideran al gurú como un Dios viviente, como una encarnación de la divinidad. Recordemos a este respecto que las más importantes religiones del mundo hablan del Hijo de Dios o del Mensajero de Dios. Por ello, muchos mahatmas son considerados como portadores de la luz divina. Un ejemplo actual es el Dalai Lama en el exilio, quien es reverenciado por miles de seres humanos como el Buda viviente. 
La llamada iniciación es un elemento esencial entre el yogui y el gurú. Este procedimiento marca el establecimiento de una relación íntima entre el gurú y el discípulo. También existen varias maneras de acceder a la iniciación. Ésta puede surgir del tacto o «sparse», esto es, cuando el gurú toca físicamente al discípulo y se establece la transmisión. También puede ser visual o «"caksusi», cuando el gurú simplemente mira al neófito. Otra manera de iniciación es de tipo mental o «manasi», en la que el gurú simplemente piensa en el discípulo y queda así iniciado el movimiento.

La importancia de las experiencias místicas es concientizar que cada momento de la vida nos acerca a la muerte, por lo que uno de los conocimientos más importantes que podemos adquirir en esta vida es aprende a morir. Ahora bien, el mukti no se alcanza de manera automática con la propia muerte debido, entre otras razones, a la teoría del karma; esto es, a las consecuencias de todos nuestros pensamientos, palabras, acciones (incluso sueños). El karma no sólo responde a la ley de acción-reacción de nuestros pensamientos, palabras y acciones presentes. En realidad, este concepto es más complejo.

Existen tres maneras de crear karma. Para explicarlo, utilizaré una alegoría. La primera acepción del concepto 'karma' se refiere a las «semillas» que en vidas pasadas hemos sembrado (en el pasado de nuestra vida actual), y de las que ahora cosechamos sus frutos. Este karma es el responsable de nuestra presente existencia y es conocido como «pralabdh». La segunda acepción se refiere a las semillas que actualmente estamos sembrando y cuyos frutos también recolectamos durante nuestra actual existencia. Este karma es conocido como «kriyaman». La tercera acepción se refiere a los frutos que todavía no han sido recolectados y que hemos «sembrado» en el pasado y que seguimos sembrando en el presente. Estos karmas están, por así decirlo, almacenados. A este karma se le conoce como «sinchiti», el cual tendrá que ser "saldado» en futuras reencarnaciones. Notemos que, de acuerdo con esta explicación, es indiscernible verificar si una acción particular es causa o efecto de un karma, esto es, no podemos saber si estamos cosechando el fruto kármico o sembrando la semilla. Así visto, estamos atrapados en el inevitable proceso kármico de sembrar y cosechar karmas. Este proceso es la principal causa de que estemos «atrapados» en la rueda de la reencarnación o samsara que nos aleja del mukti.

Por lo anterior, el concepto de karma para esta tradición yóguica es más complejo que la noción occidental que reduce este concepto a la «suerte» que una persona tiene desde antes de su nacimiento. El karma, para esta tradición yóguica, no es el destino predeterminado de cada individuo. Tampoco es la única causa de que no alcancemos el mukti. Nuestra ignorancia o «maya» (ilusión, desilusión, decepción, irrealidad) tiene un papel 
preponderante en este proceso. El maya constituye el universo fenoménico, esto es, todo lo que no es eterno, todo lo que parece que es; pero no es. Constituye el «velo» de la ilusión que nos esconde y aleja de la divinidad. Dado que ningún ser vivo está consciente de sus vidas previas, donde sea que un alma se sitúe se sentirá contento en esa condición. Es por ello por lo que todo ser vivo no quiere morir y trata de aferrarse a la vida.

Notemos que de acuerdo con el concepto de 'maya', tanto las buenas como las malas acciones nos atan a este mundo. La razón es que todas las acciones son realizadas con un motivo y este motivo es el que nos encadena. En este sentido, la vida humana es el resultado kármico de la «combinación» de buenos y malos karmas. De acuerdo con el Surat Shabdt Yoga, antes del nacimiento el alma individual habitaba un plano mental. $\mathrm{Al}$ nacer, nos conectamos con el mundo a través de las nueve puertas del cuerpo, a saber, dos ojos, dos oídos, dos fosas nasales, la boca, el órgano sexual y el orificio anal. Si recondujéramos la esparcida atención que se extiende a través de estas vías hacia todo el cuerpo y hacia fuera - hacia el mundo fenoménico - y lográramos retraerla hacia el centro del ojo que está en medio de las dos cejas y que es conocido como «tisra til» o tercer ojo; nos desconectaríamos de este plano material y nos reconectaríamos con el plano mental. De manera que cuando el yogui logra retraer su atención hacia tisra til, sólo entonces logra abandonar el plano físico y acceder al plano mental y posteriormente al plano astral. Cuando la concentración en el tercer ojo es completa, una música interna ayuda al yogui a separarse de su propio cuerpo en un proceso que va desde las plantas de los pies hasta el tercer ojo y mediante el cual accede al primer plano espiritual. Quien ha habitado en estas regiones espirituales, se afirma, no se conmueve ni de placer ni de dolor con los altibajos del mundo; logrando un tipo de felicidad ecuánime o "ananda» que es independiente del mundo fenoménico.

En resumen, de acuerdo con la tradición yóguica del Surat Shabdt Yoga, dado que estamos atrapados en el maya, nuestro espíritu está desviado por la mente, la mente por los sentidos y los sentidos por los objetos sensibles. Dicho de otro modo, los objetos controlan nuestros sentidos, los sentidos a la mente y la mente al espíritu. Es por ello por lo que el principal objetivo de la práctica yóguica es lograr una ascensión espiritual a través de la experiencia mística que revierta este proceso y permita que nuestro espíritu controle a la mente y la mente a los sentidos.

Hasta aquí el estudio de caso. Sin embargo, cabe señalarse que algo parecido a lo que se afirma en este estudio de caso también fue sostenido por algunos filósofos como Pseudo Dionisio (n. V o VI d.C.), quien le agregó un rasgo problemático a la experiencia mística, a saber, que es indescriptible: «Una vez más, ascendiendo más alto, mantenemos que Él no 
es espíritu, o mente, ni está dotado de la facultad de la imaginación, conjetura, razón o entendimiento... ni puede ser descrito por la razón o percibido por el entendimiento.» (Pseudo Dionisio, 1920, 200). Pero no sólo la experiencia mística es indescriptible, de acuerdo con Maimónides (n. 11351204) también lo es la naturaleza misma de Dios: «la descripción de Dios a partir de negaciones, aunque sea apreciado y exaltado, es la descripción correcta... Si uno lo describe a partir de afirmaciones, implica, como hemos ya dejado claro, que lo asociemos con lo que Él no es.» (Maimónides, 1963, 134). De acuerdo con Gregorio Niseno (n. 335-394), la naturaleza de Dios no sólo es indescriptible; también es inefable: «Dios es en sí mismo lo que cualquier religión considere que es, pero lo que es nombrado por aquellos que hablan acerca de Él, no es lo que en realidad Él es porque su naturaleza es inefable.» (Nyssa, 2007, 91).

Notemos que la oración que afirma que «... su naturaleza es inefable» exhibe cierto contenido proposicional, esto es, que Dios es inefable; de la misma manera que lo hace la afirmación judeo-cristiana (1) que revisamos más arriba, a saber, que Dios es omnisciente. Asimismo, existen varias oraciones que podemos extraer del estudio de caso que acabamos de revisar las cuales también exhiben cierto contenido proposicional. Por ejemplo las siguientes:

- «la reunión del individuo con la divinidad»

- «alcanzar una integración con el todo»

- «el Yo individual logra sumergirse o identificarse con el Uno absoluto»

- «encontrar la «verdad» a través de la conciencia»

- «percibir la realidad como un todo»

- «la mente del yogui no duerme y permanece atenta a las más sutiles percepciones de la conciencia»

- «el auto-descubrimiento y la purificación para poder reunirse - religarse - con Dios y lograr la liberación de los «ikas» (ataduras de la existencia)»

- «el maya constituye el universo fenoménico, esto es, todo lo que no es eterno, todo lo que parece que es; pero no es»

- «en un proceso que va desde las plantas de los pies hasta el tercer ojo y mediante el cual accede al primer plano espiritual»

Si los puntos (b) y (c) señalados en la introducción de este texto son el caso, entonces el contenido proposicional del lenguaje religioso, como se exhibe en estos ejemplos, es susceptible de ser verdadero o falso si consideramos que estas oraciones son de naturaleza descriptiva. Veamos cuál es 
el análisis que hizo Ayer de este tipo de oraciones religiosas con las que se pretende expresar y comunicar una experiencia mística.

\section{La teoría verificacionista del significado}

La teoría verificacionista del significado elaborada por Alfred J. Ayer (1936, cap. VI), representa uno de los primeros trabajos desarrollados desde la llamada "filosofía analítica» que aborda con cierto detenimiento el tema del lenguaje religioso. Sin embargo, la conclusión a la que llegó Ayer es contundente, a saber, el lenguaje religioso carece de significado. De manera que, para este autor, las oraciones que recién extrajimos del estudio de caso y que fueron utilizadas para describir la tradición yóguica del Surat Shabdt Yoga, en realidad no tienen sentido y, por ello, carecen de valor lingüístico. Esto es así porque, de acuerdo con Ayer, un enunciado descriptivo exhibe contenido factual si y sólo si es empíricamente verificable.

El principio de verificabilidad desarrollado por Ayer determina si una oración es verdadera o falsa a través de la observación directa de su contenido factual. Sin embargo, no todos los enunciados pueden ser directamente verificados por la percepción. Tal es el caso de los enunciados matemáticos y lógicos. Este tipo de enunciados, también llamados enunciados analíticos, son verdaderos en virtud del significado de sus términos constitutivos. Por ejemplo, los enunciados «todos los cuerpos son extensos» o "todos los solteros son hombres no casados», son enunciados analíticos de este tipo. Para establecer la verdad de este tipo de enunciados no necesitamos de verificación empírica alguna. De manera que, de acuerdo con Ayer, para que un enunciado descriptivo de cualquier tipo tenga significado debe ser analíticamente verdadero o empíricamente verificable. Y dado que los enunciados utilizados en el lenguaje religioso no son analíticamente verdaderos ni existe una observación concebible que los verifique o refute, no son ni verdaderos ni falsos; simplemente carecen de significado. Notemos que de acuerdo con la postura verificacionista, el problema que enfrenta el lenguaje religioso no es que sea falso; sino que no es significativo. Y no lo es en un sentido específico, carece de significado asertivo.

Un problema general que enfrentó la teoría verificacionista es que su propio criterio verificacionista no es aplicable a sí misma, esto es, la teoría verificacionista no es ni analíticamente verdadera ni empíricamente verificable, de manera que, estrictamente hablando, es una teoría que carece de significado, autorefutándose. Sin emnbargo, en lo que sigue mostraré que la teoría verificacionista no sólo se auto refuta; sino que también exhibe ciertas inconsistencias lógicas, empíricas y cognitivas que la debi- 
litan como un buen criterio para evaluar la significatividad del lenguaje religioso. Comenzaré mi análisis con los problemas de tipo lógico.

1. La paradoja de Hempel.

De acuerdo con Carl Hempel (1945), el enunciado:

(i) «todos los cuervos son negros».

puede ser entendido como implicando que si algo es un cuervo, entonces es negro. Si esto es así, (i) es lógicamente equivalente al siguiente enunciado:

(ii) «si algo no es negro, entonces no es un cuervo».

Desde el punto de vista de la lógica clásica, en todas las circunstancias en que (ii) es verdadero, (i) también es verdadero. Ahora bien, todo enunciado universal como (i) obtiene una evidencia positiva vía un enunciado observacional particular de la misma clase como el siguiente:

(iii) «este cuervo es negro».

De acuerdo con Hempel, si aplicamos esta misma regla al enunciado (ii), surge una paradoja. Veamos por qué. Si formulamos un enunciado observacional particular como el siguiente:

(iv) «esta manzana roja no es negra y no es un cuervo».

el enunciado (iv) constituye una evidencia positiva para el enunciado (ii). Pero dado que se ha establecido que el enunciado (ii) es lógicamente equivalente al enunciado (i), esto implica que observar una manzana roja también es evidencia positiva para el enunciado (i), esto es, que todos los cuervos son negros. Y ciertamente, parece extraño (al menos intutivamente) que observar una manzana roja nos proporcione información relevante acerca del color de los cuervos. Si el principio de verificabilidad de Ayer se fundamenta, como sostiene, en la observación; este problema lógico para representar un reto para su teoría.

2. El problema Inductivo.

La teoría verificacionista desarrollada por Ayer, presupone buscar evidencia empírica a favor o en contra de las afirmaciones religiosas. Siguiendo con nuestro ejemplo de los cuervos, para responder a la pregunta ¿de qué color son los cuervos?, encontrar un cuervo color negro supone evidencia positiva a nuestra pregunta. De manera que nuestra búsqueda de evidencia toma la forma de un argumento inductivo como el siguiente:

¿De qué color son los cuervos?

Cuervo 1 es negro.

Cuervo 2 es negro.

Cuervo 3 es negro... 

ficar).

Cuervo «n» es negro (donde «n» representa un número por especi-

A partir de esta evidencia, si la muestra es «suficientemente» amplia, podríamos estar tentados a concluir lo siguiente:

Por lo tanto: todos los cuervos son negros.

Sabemos que esto es incorrecto por varias razones. La primera es que ninguna confirmación particular puede derivar en una confirmación universal. Esto es, la suma de "n» enunciados observacionales particulares no implica ningún enunciado con un cuantificador universal como «todos», «siempre», "ninguno» o «nunca». Así que por muy amplia que sea la muestra, no se sigue de manera lógica que hemos confirmado que «todos» los cuervos son negros. La conclusión de un argumento inductivo de la forma anterior, sólo nos conduce a resultados probables. Esto es, la verficación perceptual sólo nos arroja resultados probables y no absolutos.

3. El problema inductivo «n $+1 »$.

Supongamos que hemos llegado a un acuerdo y que hemos registrado una cantidad «suficiente» de evidencia empírica, esto es, que hemos llegado a la evidencia «n» deseada. La lógica nos indica que aquella evidencia que no alcanzamos a registrar porque precisamente detuvimos nuestra indagatoria en «n» con el objetivo de inferir nuestra conclusión inductiva (después de todo somos seres finitos), representa lo que se conoce como la evidencia «n + 1» que ya no observamos. Siguiendo con nuestro ejemplo de los cuervos, nada nos impide pensar que ese cuervo que no registramos puede ser un cuervo que no es negro. Lo que nos impide concluir, nuevamente, que todos los cuervos son negros.

Ahora consignaré algunos argumentos de carácter empírico que, en conjunto con los anteriores argumentos lógicos, socavan aún más la teoría verificacionista desarrollada por Ayer.

4. El proceso de verificación de un enunciado observacional depende del grado de precisión de los métodos e instrumentos de prueba utilizados. Después de todo, podemos considerar como instrumentos a los órganos humanos con los que realizamos la percepción sensorial. Dado que el grado de precisión de estos métodos e instrumentos ciertamente han mejorado con el tiempo, basta considerar el avance tecnológico que hemos alcanzado en nuestros días, de aquí se sigue que el proceso de verificación empírica es un procedimiento instrumental y metodológicamente perfectible. Si esto es así, la noción de verificación es graduada y nunca es definitiva.

5. La percepción humana, como la observación, puede estar dirigida no sólo por restricciones tecnológicas y metodológicas, como se establece en el punto anterior. También por habilidades y límites cognitivos, ex- 
pectativas e intereses teóricos; incluso, por asuntos extra empíricos como son asuntos políticos, de fama, de dinero, etc. De manera que, desde este punto de vista, no puede haber una observación totalmente segura, libre del peligro de las «malas» o «erróneas» interpretaciones.

Finalmente, revisaré un argumento de naturaleza cognitiva que también socava a la teoría verificacionista.

6. Para poder verificar de manera "neutral» un enunciado observacional, al parecer requerimos de un acceso independiente a ambos dominios, esto es, al enunciado a verficar y a la evidencia que verifica. A este argumento se le ha llamado el argumento del «ojo de Dios». Irónicamente, sólo un ser metafísico con la capacidad de poseer el ojo de Dios, podría tener acceso a este punto de vista privilegiado.

En resumen, para que un enunciado religioso tenga significado debe ser analíticamente verdadero o empíricamente verificable, según el criterio verificacionista. Y dado que los enunciados utilizados en el lenguaje religioso no son analíticamente verdaderos, esto es, no son verdaderos en virtud del significado de sus términos constitutivos, entonces, de acuerdo con esta postura, deben ser enunciados de naturaleza empírica. Que los enunciados religiosos efectivamente sean enunciados de naturaleza empírica, es una cuestión abierta que no vamos a abordar aquí. Lo que hemos intentado mostrar a partir de los anteriores argumentos es que el principio de verificabilidad propuesto por Ayer no sólo se auto refuta; sino que exhibe ciertas inconsistencias lógicas, empíricas y cognitivas que lo debilitan como un buen criterio para evaluar la significatividad del lenguaje religioso.

\section{Conclusiones}

Los argumentos de tipo lógico, empírico y cognitivo que desarrollamos muestran que el principio de verificabilidad propuesto por Ayer no sólo se auto refuta; sino que exhibe ciertas inconsistencias que lo debilitan como un buen criterio para evaluar la significatividad del lenguaje religioso. De acuerdo con nuestro análisis, dicho principio es un procedimiento inacabado e inconcluso desde un punto de vista lógico; sesgado y perfectible desde un punto de vista empírico e inaccesible, desde un punto de vista cognitivo.

De nuestro análisis se desprende que no existe una frontera natural entre un enunciado descriptivo y el contenido factual que supuestamente se verifica. De acuerdo con nuestro argumento, los instrumentos y métodos de verificación están limitados por nuestras habilidades, expec- 
tativas e intereses cognitivos específicos; por lo que el valor de verdad del contenido fáctico de los enunciados religiosos que revisamos en nuestro estudio de caso, no puede ser decidido de forma indubitable, esto es, ningún enunciado fáctico puede ser verificado definitivamente mediante prueba empírica alguna. Si esto es así, una línea abierta de investigación es investigar si los enunciados observacionales pertenecientes a cualquier tipo de lenguaje, entre éstos el lenguaje religoso, pueden ser derivados a partir de otros enunciados observacionales y no a partir de su contenido fáctico.

\section{Bibliografía}

Ayer, Alfred. Language, Truth and Logic, New York, Dover Publications, 1936.

Gellman, Jerome. «Mysticism and Religious Experience». En William J. Wainwright (ed.) The Oxford Handbook of Philosophy of Religion, Oxford, Oxford University Press (2005), pp. 138-167.

Hempel, Carl. «Studies in the Logic of Confirmation». Mind, Vol. 54, No. 213, (1945), pp. 1-26.

Maimonides, Moses. The Guide of the Perplexed. Chicago, University of Chicago Press, 1963.

Martin, Michael. «The Verificationist Challenge». En Charles Taliaferro et al (eds.) A Companion to Philosophy of Religion, Sussex, Blackwell Publishing (2010), pp. 458-466.

Nyssa, Gregory. Contra Eunomium II, Leiden, Brill, 2007.

Pseudo Dionisio. On The Divine Names and The Mystical Theology, New York, The Macmillan Company, 1920.

Soami, Hazur. Sar Bachan. Amritsar, Radha Soami Satsang Beas, 1983.

Wierenga, Edward. «Philosophy of religion». En John V. Canfield (ed.) Philosophy of Meaning, Knowledge and Value in the Twentieth Century, London, Routledge (1997), pp. 292-304. 
$-54-$ 